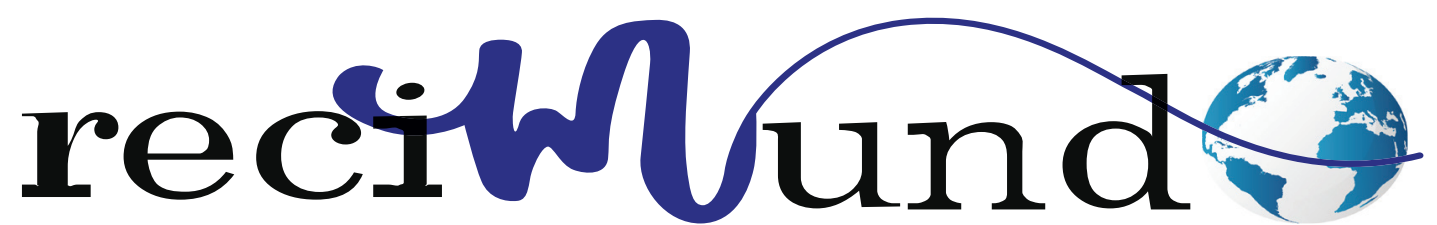

Revista Científica Mundo de la Investigación y el Conocimiento

DOI: 10.26820/recimundo/4.(1).esp.marzo.2020.58-64

URL: http://recimundo.com/index.php/es/article/view/776

EDITORIAL: Saberes del Conocimiento

REVISTA: RECIMUNDO

ISSN: 2588-073X

TIPO DE INVESTIGACIÓN: Artículo de Revisión

CÓdIGO UNESCO: 3201 Ciencias Clínicas

PAGINAS: 58-64

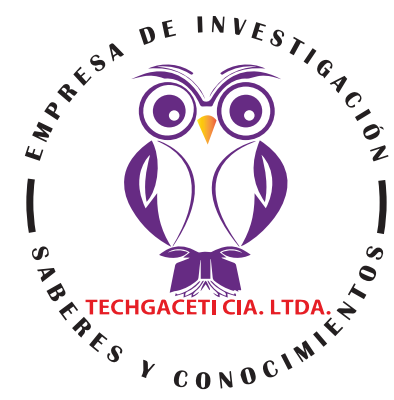

\title{
Indicaciones de Hemorroidectomía
}

\author{
Hemorrhoidectomy indications
}

\section{Indicações de Hemorrhoidectomy}

Andrés Fernando Alcocer Ortega ${ }^{1}$; Aracely Vanessa Aguilar Cobo²; Carlos Eduardo Fernández Cuenca;

Andrés Fabricio Huilca Ortiz ${ }^{4}$

RECIBIDO: 20/11/2019 ACEPTADO: 29/01/2020 PUBLICADO: 05/03/2020

1. Médico; Investigador Independiente; Quito, Ecuador; af_andres-fernandomed@hotmail.com; (D) https://orcid. org/0000-0003-4172-6601

2. Médico; Investigadora Independiente; Quito, Ecuador; aracelyv19@hotmail.com; (D) https://orcid.org/00000002-9489-8012

3. Médico; Investigador Independiente; Quito, Ecuador; carlitosfc@gmail.com; (D) https://orcid.org/0000-00021116-6714

4. Máster Universitario en Dirección y Gestión Sanitaria; Médico Cirujano; Investigador Independiente; Quito, Ecuador; andresh_316@hotmail.com; (D) https://orcid.org/0000-0002-5062-9259

CORRESPONDENCIA

Andrés Fernando Alcocer Ortega

af_andres-fernandomed@hotmail.com

Quito, Ecuador 


\section{RESUMEN}

Las hemorroides o almorranas son la patología más frecuente en el campo de la coloproctología y afectan a millones de personas en todo el mundo, son estructuras fisiológicas constituidas por plexos arteriovenosos que forman un almohadilla a lo largo del conducto anal. Asociadas a los componentes musculares y el tejido epitelial situado en el canal anal, sirven para mantener la continencia anal durante los periodos no defecatorios. El tratamiento de las hemorroides es aplicado de acuerdo a la sintomatología que presente el paciente y a la progresión de la misma. El manejo de las hemorroides puede ir desde el tratamiento conservador al quirúrgico. El enfoque general del presente trabajo ha sido el del tratamiento quirúrgico conocido como hemorroidectomía, y las indicaciones para el cuidado postoperatorio. Los resultados obtenidos mediante el desarrollo de una investigación de tipo bibliográfica, delimitada a una metodología de revisión, sirvieron para alcanzar los objetivos preestablecidos, y adicionalmente conocer sus tipos y complicaciones más comunes. En conclusión, el referido tratamiento es una de las intervenciones quirúrgicas más frecuentes en el área de proctología, con excelentes resultados en la corrección de hemorroides grado III y IV y aquellas cuyos tratamientos convencionales no fueron efectivos. En el caso de la hemorroidectomía, la mayoría de los cuidados recaen directamente sobre el paciente y en todo caso, sobre algún cuidador o colaborador familiar, ya que el alta suele darse el mismo día o al día siguiente, por lo que fundamental es que el cirujano informe ampliamente al paciente sobre qué esperar después de la operación y cuáles son los signos o síntomas a los que debe prestar mayor atención para comunicárselos y evitar complicaciones.

Palabras clave: Hemorroides, Cirugía, Hemorroidectomía, Resección, Hemorragia.

\section{ABSTRACT}

Hemorrhoids or piles are the most common pathology in the field of coloproctology and affect millions of people around the world, they are physiological structures made up of arteriovenous plexuses that form a pad along the anal canal. Associated with muscle components and epithelial tissue located in the anal canal, they serve to maintain anal continence during non-defecatory periods. Hemorrhoids treatment is applied according to the symptoms presented by the patient and the progression of the same. Hemorrhoid management can range from conservative to surgical treatment. The general focus of this work has been the surgical treatment known as hemorrhoidectomy, and the indications for postoperative care. The results obtained through the development of a bibliographic research, delimited to a review methodology, served to achieve the pre-established objectives, and additionally to know their most common types and complications. In conclusion, the aforementioned treatment is one of the most frequent surgical interventions in the area of proctology, with excellent results in the correction of hemorrhoids grade III and IV and those whose conventional treatments were not effective. In the case of hemorrhoidectomy, most of the care falls directly on the patient and in any case, on a family caregiver or collaborator, since discharge is usually the same day or the next day, so it is essential that the Surgeon extensively inform the patient about what to expect after the operation and what are the signs or symptoms to which he should pay more attention to communicate them and avoid complications.

Keywords: Hemorrhoids, Surgery, Hemorrhoidectomy, Resection, Hemorrhage.

\section{RESUMO}

Hemorróidas ou pilhas são a patologia mais comum no campo da coloproctologia e afetam milhões de pessoas em todo o mundo, são estruturas fisiológicas compostas por plexos arteriovenosos que formam uma almofada ao longo do canal anal. Associados a componentes musculares e tecido epitelial localizados no canal anal, servem para manter a continência anal durante períodos não defecatórios. O tratamento de hemorróidas é aplicado de acordo com os sintomas apresentados pelo paciente e a progressão do mesmo. O tratamento da hemorróida pode variar de tratamento conservador a cirúrgico. O foco geral deste trabalho tem sido o tratamento cirúrgico conhecido como hemorrhoidectomy, e as indicações para cuidados pós-operatórios. Os resultados obtidos com o desenvolvimento de uma pesquisa bibliográfica, delimitada por uma metodologia de revisão, serviram para atingir os objetivos pré-estabelecidos e, adicionalmente, para conhecer os tipos e complicações mais comuns. Concluindo, o tratamento mencionado é uma das intervenções cirúrgicas mais frequentes na área da proctologia, com excelentes resultados na correção das hemorróidas grau III e IV e naquelas cujos tratamentos convencionais não foram eficazes. No caso de hemorrhoidectomy, a maioria dos cuidados recai diretamente no paciente e, em qualquer caso, em um cuidador familiar ou colaborador, uma vez que a alta é geralmente no mesmo dia ou no dia seguinte, por isso é essencial que o Cirurgião informe extensivamente o paciente sobre o que esperar após a operação e quais são os sinais ou sintomas aos quais ele deve prestar mais atenção para comunicá-los e evitar complicações.

Palavras-chave: Hemorróidas, Cirurgia, Hemorrhoidectomy, Ressecção, Hemorragia. 


\section{Introducción}

Las hemorroides o almorranas son una patología muy común y conocida en el campo de la Coloproctología y afecta a millones de personas en todo el mundo. Esta patología es considerada como uno de los trastornos más comunes de la población en general y una de las principales causas de rectorragia en atención primaria. "Su prevalencia, en general, es difícil de precisar, pues existen pacientes que, por padecer síntomas leves, o bien por pudor, no consultan esta patología". (Puga, 2017)

Las hemorroides son muy comunes en personas de ambos sexos. Se calcula que la mitad de las personas en el mundo tienen hemorroides a los 50 años. "El síntoma más común de las hemorroides dentro del ano es sangre roja brillante en las heces, en el papel higiénico o en el escusado. Los síntomas suelen desaparecer en algunos días". (Enciclopedia Médica ADAM, 2019).

En estudio publicado en Mayo Clinic (2019) mencionan las siguientes complicaciones:

- Anemia. En raras ocasiones, la pérdida crónica de sangre a causa de las hemorroides puede causar anemia, en la cual no tienes suficientes glóbulos rojos saludables para transportar oxígeno a las células.

- Hemorroide estrangulada. Si se interrumpe el suministro de sangre a una hemorroide interna, la hemorroide puede ser "estrangulada", lo que puede causar dolor extremo.

- Coágulo sanguíneo. Ocasionalmente, se puede formar un coágulo en una hemorroide (hemorroide trombosada). Aunque no es peligroso, puede ser extremadamente doloroso y a veces necesita ser punzado y drenado.

"La Hemorroidectomía quirúrgica está indicada en los pacientes que no responden a otras formas de tratamiento y en aquellos pacientes con hemorroides internas grado IV. El dolor posoperatorio significativo es frecuente, así como la retención urinaria y el estreñimiento". (Ansari, 2018)

A pesar del intenso dolor postoperatorio, esta cirugía presenta beneficios tales como ser de tipo ambulatoria, donde el paciente probablemente volverá a su casa el mismo día o al siguiente, y con mejores resultados a largo plazo, para quitar las hemorroides. Es por ello que los primeros cuidados que se presten al paciente operado y la información y cuidados del paciente en el hogar, son parte fundamental de la recuperación rápida y el mejor confort del paciente durante el proceso.

El propósito fundamental de la presente investigación es plasmar todo lo relacionado con los Indicaciones de la Hemorroidectomía, incluyendo concepto, tipos y sus principales complicaciones, a los efectos de una mejor comprensión del objeto general.

\section{Materiales y Métodos}

La revisión que se presenta se limitó al hallazgo de diversos tipos de materiales bibliográficos disponibles completa y abiertamente, entre los que se escogieron: artículos científicos, guías clínicas, ensayos clínicos, estudios o reportes de casos, tesis de grado, posgrado y doctorado, boletines $\mathrm{y} / \mathrm{o}$ folletos de instituciones oficiales o privadas de reconocida trayectoria en el área de la salud, medicina o cientificoacadémica, y demás, monografías u otros tipos de documentos que, a criterio del equipo, mostraran información de interés en base a la observación y evaluación de la evidencia mostrada en sus contenidos, representando este proceso en una serie de resultados que oscilaron entre 5 y 62 enlaces a fuentes de información bibliográficas.

Algunas de las bases de datos consultadas que rindieron mejores resultados fueron: la página oficial de la Organización Mundial 
de la Salud (OMS), Mayo Clinic, SciELO, MedlinePlus, entre otras.

Los términos de búsqueda que se utilizaron fueron "hemorroidectomía" y "tratamiento quirúrgico de las hemorroides", a los que se le aplicaron criterios de selección tales como: idioma español (e inglés en algunos casos); publicación entre 2010 y 2020 (ambos inclusive), salvo algunas excepciones; acceso completo y abierto; en materia de salud y medicina; estudios referidos a humanos; entre algunos otros.

Motivado a los hallazgos y para fines del objetivo general de este artículo, la información se organizó y discutió haciendo énfasis en: las opciones de cuidados postoperatorios para pacientes sometidos a una hemorroidectomía y aspectos asociados a esta condición, tales como: conceptos básicos, tipos y complicaciones de la hemorroidectomía.

\section{Resultados}

Las hemorroides son estructuras fisiológicas constituidas por plexos arteriovenosos que forman un almohadilla a lo largo del conducto anal. Asociadas a los componentes musculares y el tejido epitelial situado en el canal anal, sirven para mantener la continencia anal durante los periodos no defecatorios.

En la práctica tiende a emplearse el término de hemorroides cuando estas venas se dilatan convirtiéndose en varicosidades, que pueden protruir o trombosarse, causando dolor, o ulcerarse, produciendo sangramiento. "La enfermedad hemorroidal constituye la patología proctológica más frecuente y una de las principales razones de consulta médica. El 5 \% de la población mundial presenta síntomas relacionados con ellas y la prevalencia alcanza el $50 \%$ a partir de los 50 años de edad." Hernández et al. (2014).

El tratamiento de las hemorroides es apli- cado de acuerdo a la sintomatología que presente el paciente y a la progresión de la misma. El manejo de las hemorroides puede ir desde el tratamiento conservador al quirúrgico.

La cirugía para las hemorroides constituye la última opción cuando los tratamientos conservadores no han resultado exitosos o cuando la gravedad de la enfermedad así lo requiere. De acuerdo a lo indicado por la (Enciclopedia Médica ADAM, 2019) la cirugía puede involucrar:

- Colocar una pequeña liga de caucho alrededor de una hemorroide para encogerla al bloquear el flujo de sangre.

- Engrapar una hemorroide para bloquear el flujo de sangre, provocando que se encoja.

- Utilizar una navaja (bisturí) especial para extirpar hemorroides. Usted puede o no recibir suturas.

- Inyectar un químico a un vaso sanguíneo de la hemorroide para encogerla.

- Utilizar un láser para quemar la hemorroide.

\section{Tipos}

En estudio publicado en (Quirúrgica, 2019) refieren que el tratamiento quirúrgico de las hemorroides, puede ser llevado a cabo mediante las siguientes técnicas:

- Cirugía convencional: con bisturí eléctrico monopolar.

- Con láser: en especial en aquellas hemorroides con predominio de componente externo. El láser, sin que haya demostrado que acorte el tiempo de cicatrización de las heridas, produce una menor y más controlada lesión térmica en los tejidos. Esto se traduce en menor inflamación postoperatoria y, por tanto, menos dolor y más rápida reincorporación a las actividades habituales.

- Con ligasure: sellador de vasos y tejidos monopolar. 
- Técnica de Longo o mucosectomía con grapadora mecánica: Con esta técnica se elimina el prolapso hemorroidal y mucoso mediante la resección circunferencial de una banda de mucosa rectal, quedando la herida a un nivel interno menos doloroso que se traduce en un postoperatorio más confortable.

- THD (desarterialización hemorroidal transanal): consiste en suturar con puntos quirúrgicos la arteria hemorroidal que lleva el flujo de sangre a los paquetes hemorroidales.

Por su parte De Miguel et al. (2005) Refieren que hay diferentes técnicas de hemorroidectomía, según sigue:

La abierta de Milligan-Morgan, la cerrada de Ferguson, la submucosa de Parks y la circunferencial de Whitehead. Hay estudios que han comparado la hemorroidectomía abierta con la cerrada, sobre todo con el objetivo de valorar si hay diferencias en el dolor postoperatorio. En uno de los primeros estudios aleatorizados, en que se comparaba la técnica abierta con la submucosa de Parks, no se encontraron diferencias en las escalas de dolor postoperatorio, e incluso se describieron amplios rangos de dolor en ambos grupos. Sí refirió encontrar una mejor sensibilidad anal medida mediante técnica de electrosensibilidad mucosa en el grupo de hemorroidectomía submucosa, que sin embargo no se refleja clínicamente al no encontrar diferencias en la función defecatoria, tanto en la discriminación como en las alteraciones de la continencia anal. Sin embargo, otro estudio aleatorizado en que se compara también la técnica de MiIligan-Morgan con la submucosa de Parks, durante las primeras 2 semanas después de la cirugía, encuentra que el grupo de la técnica submucosa tiene menos dolor en el momento de la defecación y después de ella, menos dolor al sentarse, menos prurito y menos ensuciamiento. Igualmente, el grupo de técnica submucosa tiene una menor estancia en el hospital y una menor incapa- cidad laboral.

\section{Complicaciones}

De acuerdo a lo indicado por Rubio (2017) las complicaciones de la hemorroidectomía serían:

- Retención urinaria.

- Hemorragia (inmediata: por mala hemostasia; o tardía: alrededor del $7^{\circ}$ día, por caída de la escara, que cede naturalmente en la mayoría de los casos).

- Absceso de la herida.

- Estenosis, por puentes mucosos insuficientes.

- Incontinencia: transitoria en la mayoría de los casos; mientras que en ancianos por lo general es definitiva por la dilatación anal excesiva.

- Plicomas, por no resecar correctamente la piel perianal redundante.

\section{Cuidados postoperatorios}

Más de un 90\% de las cirugías de hemorroides son exitosas. Sin embargo, se considera una cirugía con un postoperatorio muy doloroso, por lo cual la atención y cuidados que se brinde al paciente resultan de vital importancia para la recuperación del mismo. Los medicamentos para el dolor y reblandecedores de materia fecal, son indispensables los primeros días, con la finalidad de reducir el malestar y evitar el esfuerzo durante las evacuaciones intestinales. El tiempo de recuperación varía de un paciente a otro, al tipo de hemorroides, al procedimiento quirúrgico utilizado, al umbral del dolor del paciente y a otras patologías presentes, entre otros, no obstante, es posible la recuperación total en dos semanas aproximadamente.

\section{Cuidados generales}

Una vez dado de alta, lo más probable y molesto que el paciente puede sentir es el dolor, que va de medio a intenso, para lo 
cual el paciente debe tomar la medicación (analgésicos) prescrita por el médico tratante, es importante tomarlos a la hora indicada y no esperar a que el dolor se vuelva intenso para tomarlo. Asimismo, el paciente puede presentar un poco de sangrado, especialmente después de la primera deposición, por lo que se recomienda dieta suave, sobre todo durante los primeros días posteriores a la cirugía. Es importante, tomar muchos líquidos, como caldo, jugos, y agua. También el médico puede sugerir el uso de un ablandador de heces. En cuanto al cuidado de la herida es recomendado el uso de una gasa o una toalla sanitaria con la finalidad de absorber cualquier secreción de la herida y cambiarla con frecuencia. Con respecto a la actividad física, es recomendado regresar gradualmente a las actividades normales, evitar actividades extenuantes hasta tanto la zona de la cirugía esté completamente sana, incluyendo el esfuerzo durante la defecación o la micción. Se debe incrementar el nivel de actividad física a medida que el paciente se vaya recuperando.

Igualmente, dentro de los cuidados se puede incluir la aplicación de compresas de hielo en la zona de la cirugía a los fines de la reducción de la hinchazón y el dolor, envolviendo el hielo en un paño o toalla limpia con anterioridad y sin exceder 15 minutos a la vez, para proteger la zona y no producir heridas en la piel.

Asimismo, debe tomarse en cuenta los cuidados básicos de la enfermedad para una mejor evolución en el postoperatorio, tales como: mantener una dieta equilibrada, principalmente rica en fibras, tomar laxante en caso de que se requiera, fomentar la ejercitación gradual, comenzando con paseos diarios, es importante evitar el tabaco, usar ropa cómoda y transpirable, evitar el consumo de alcohol y tomar abundantes líquidos, unos 1.5 litros de agua al día.

\section{Manejo del dolor postoperatorio}

Uno de los principales síntomas esperados en cualquier cirugía es el dolor. Los médicos deben prescribir al paciente la medicación acorde al nivel de dolor percibido y a las necesidades propias de su condición, en todo caso, lo propio para obtener una recuperación rápida del paciente es brindarle la mayor satisfacción durante este procedimiento.

En estudio publicado por Roig \& García (2012) los cuidados postoperatorios farmacológicos para el manejo del dolor, incluyen:

- Se pueden emplear la analgesia epidural o la analgesia controlada por el paciente (PCA), pero no son opciones adecuadas en cirugía ambulatoria.

- Una alternativa es el empleo de opiáceos por vía subcutánea o intramuscular. El ketorolaco se ha aconsejado también, tanto intraesfinteriano como parenteral.

- En nuestro medio es más habitual emplear, a partir de las 6-12 horas de la operación, analgesia oral con: metamizol, tramadol, etc., asociada a antiinflamatorios. También se han recomendado ansiolíticos y relajantes musculares. Existe alguna evidencia de que el empleo de metronidazol oral disminuye el dolor entre el $2^{\circ}$ y $4^{\circ}$ día. Se han empleado también la nitroglicerina tópica al $0,2 \%$ y la fracción flavonoide micronizada, que ayuda a disminuir el edema.

\section{Conclusiones}

La hemorroidectomía es una de las intervenciones quirúrgicas más frecuentes en el área de proctología, con excelentes resultados en la corrección de hemorroides grado III y IV y aquellas cuyos tratamientos convencionales no fueron efectivos.

Los cuidados postoperatorios tienen como propósito mantener al paciente sano y evitar cualquier tipo de complicaciones. En el

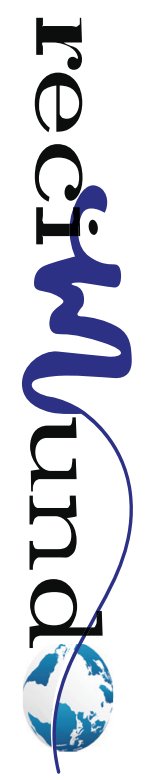


caso de la hemorroidectomía, dado que se refiere a una cirugía ambulatoria, la mayoría de los cuidados recaen directamente sobre el paciente y en todo caso, sobre algún cuidador o colaborador familiar, ya que el alta suele darse el mismo día o al día siguiente. Para lo cual es fundamental que el cirujano informe ampliamente al paciente sobre qué esperar después de la operación y cuáles son los signos o síntomas a los que debe prestar mayor atención para comunicárselos y evitar complicaciones.

Uno de los aspectos negativos de la hemorroidectomía es el dolor postoperatorio por lo que es de gran importancia que los profesionales de la salud prescriban medicamentos y pasos a seguir en el hogar para minimizar este síntoma. Otro aspecto fundamental a considerar es la higiene de la herida la cual debe permanecer seca y limpia. Asimismo, es importante mantener las heces suaves ya sea con una dieta específica o con el uso de ablandadores. Por último, la incorporación gradual a la actividad física y laboral del paciente puede influir tanto biológica como psicológicamente en su recuperación. En su conjunto, todos estos cuidados proporcionarán el confort necesario al paciente para evitar cualquier tipo de complicación que se pueda derivar de la operación y permitirá que el paciente se sane en un menor periodo de tiempo.

\section{Bibliografía}

Ansari, P. (Julio de 2018). Manual MSD. Recuperado el 31 de 01 de 2020, de https://www. msdmanuals.com/es-es/professional/trastornos-gastrointestinales/trastornos-anorrectales/hemorroides?query=hemorroides
De Miguel, M., Oteiza, F., Ciga, M., \& Ortiz, H. (12 de 2005). Tratamiento quirúrgico de las hemorroides. 78(S3), 15-23. Recuperado el 06 de 02 de 2020, de https://www.elsevier.es/es-revista-cirugia-espanola-36-articulo-tratamiento-quirurgico-las-hemorroides-13083394

Enciclopedia Médica ADAM. (07 de Marzo de 2019). MedlinePlus. Recuperado el 03 de 02 de 2020, de https://medlineplus.gov/spanish/hemorrhoids.html

Enciclopedia Médica ADAM. (27 de Mayo de 2019). MedlinePlus. Recuperado el 04 de 02 de 2020, de https://medlineplus.gov/spanish/ency/article/002939.htm

Hernández, F., Valenzuela, C., Catasús, K. M., Lazo, O., Hernández, R., Bermúdez, C. H., . . . Faríñez, A. (2014). Caracterización epidemiológica de la enfermedad hemorroidal aguda en hospitales seleccionados en Cuba (noviembre de 2011 a enero 2012). Revista Cubana de Higiene y Epidemiología, 52(1), 29-43. Recuperado el 02 de Enero de 2020, de http://scielo.sld.cu/pdf/hie/v52n1/ hig04114.pdf

Mayo Clinic. (14 de Diciembre de 2019). Mayo Clinic. Recuperado el 06 de 02 de 2020, de https:// www.mayoclinic.org/es-es/diseases-conditions/ hemorrhoids/symptoms-causes/syc-20360268

Puga, L. (2017). Hemorroides. Servicio de Atención Primaria. Servicio Galego de Saúde. Lugo. España. Recuperado el 30 de 01 de 2020, de https:// www.fisterra.com/guias-clinicas/hemorroides/

Quirúrgica. (20 de 12 de 2019). Quirúrgica. Recuperado el 06 de 02 de 2020, de quirurgica.com: https://www.quirurgica.com/sobre-quirurgica-2/

Roig, J., \& Garcia, J. (2012). Guía de Práctica Clínica: Hemorroides. Recuperado el 30 de 01 de 2020, de http://ucpvalencia.es/wp-content/ uploads/2015/10/Gu\%C3\%ADa-de-cuidados-Hemorroides.pdf

Rubio, U. (2017). Técnica Quirúrgica Hemorroidectomía. Diapositivas, Betania Especialidades Médicas. Recuperado el 06 de 02 de 2020, de https:// es.slideshare.net/BetaniaEspecialidadesMdicas/ tcnica-quirrgica-hemorroidectoma

\section{CITAR ESTE ARTICULO:}

Alcocer Ortega, A., Aguilar Cobo, A., Fernández Cuenca, C., \& Huilca Ortiz, A. (2020). Indicaciones de Hemorroidectomía. RECIMUNDO, 4(1(Esp)), 58-64. doi:10.26820/recimundo/4.(1).esp.marzo.2020.58-64

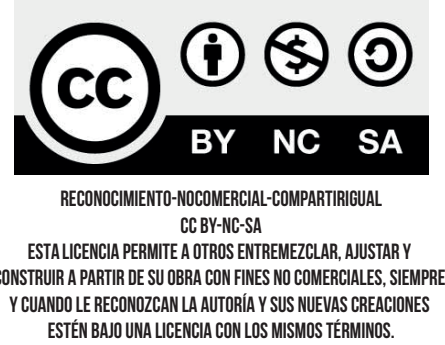

